\title{
Motivación, Universidad de Calidad y Servicios de Counseling en la Universidad de Granada
}

\section{José Luis Arco Tirado ${ }^{1}$, Francisco D. Fernández Martín ${ }^{1}$, Sagrario López Ortega ${ }^{2}$ y Verónica Heilborn Díaz}

\footnotetext{
${ }^{1}$ Departamento de Psicología Evolutiva y de la Educación, Universidad de Granada, ${ }^{2}$ Gabinete Psicopedagógico, Universidad de Granada
}

Granada, España

jlarco@ugr.es 


\section{Resumen}

Introducción. Los datos evaluadores sobre rendimiento y abandono de los estudios en el ámbito universitario español, justifican el momento de cambios estructurales, organizativos y curriculares que vive la Universidad española. El estudio de los problemas de motivación en los estudiantes de la universidad, a la base de muchos de estos problemas, se presenta como objetivo prioritario. Sin embargo, la complejidad del fenómeno, unido a las limitaciones de los modelos psicológicos disponibles, acentúa la necesidad de más investigación, especialmente aplicada en este terreno.

Método. Los sujetos que componen nuestra muestra acuden al servicio del Gabinete Psicopedagógico (GPP) por voluntad propia. Para evaluar y atender sus demandas se les aplica un protocolo de información psicopedagógico en el que recogemos los datos de tipo demográfico, psicológico, académico y vocacional-profesional que presentamos en este estudio.

Resultados. Los resultados muestran los porcentajes y proporciones de estudiantes con problemas de motivación que subyacen a la conducta de búsqueda de este tipo de servicios. Otros resultados recogidos, completan y corroboran la potencialidad de algunos de los modelos actuales para mejorar la potencia y la utilidad de las futuras intervenciones.

Discusión. La volatilidad del fenómeno de la motivación en humanos quizás obedezca a las limitaciones de los modelos teóricos disponibles hasta el momento. De ahí quizás la necesidad de medir y recoger datos en los ámbitos cognitivo y afectivo como codeterminantes de los niveles de motivación de los estudiantes. Los datos aportados por este estudio permiten corrobaran la validez y utilidad del modelo aquí contrastado y lo recomiendan para futuras intervenciones.

Palabras Clave: motivación, rendimiento académico, abandono de laUniversidad, retención, desesperanza aprendida, indefensión aprendida, compañeros, experiencias escolares, feedback negativo de profesores. 


\section{Introducción}

La Convergencia Europea de la Educación Superior (p.e. Acuerdo de Bolonia), la reforma del sistema de acceso del profesorado a la función pública docente en la Universidad (p.e. LOU) o la modificación del sistema de acceso a la Universidad para estudiantes de Bachillerato, son tres ejemplos claros de los cambios que están modificando el panorama universitario español. A promover estos cambios ha contribuido la pobre cuenta de resultados que presenta la universidad en sus tres funciones básicas como son la formación, la investigación y el cambio social (Arco-Tirado y Fernández-Balboa, 2003). En este sentido, Bricall (2000) señala la escasez de datos procedentes de investigaciones evaluadoras que amparen y justifiquen los cambios propuestos y adoptados en la universidad. Por otra parte Vidal, Díez y Vieira (2001) llaman la atención sobre el hecho de que ninguna de las dos reformas legislativas universitarias propuestas en los últimos 20 años (LRU, 1983; LOU, 2001), incluye análisis o previsiones dirigidas a crear y desarrollar servicios que atiendan las necesidades psicopedagógicas del alumnado que provocan su desadaptación a las exigencias de la vida universitaria.

En medio de todos estos complejos procesos de transformación y cambio se encuentra el alumnado, colectivo sobre el cual la situación anteriormente descrita ejerce un gran impacto, tal y como manifiestan los datos de evaluaciones recientes disponibles que indican que el $37 \%$ de los alumnos de ciclo largo cumple con éxito la trayectoria, el $31 \%$ acumula retraso y el $32 \%$ abandona, siendo en ciclo corto el 53,5\%, 25\% y 22\%, respectivamente (Consejo de Universidades, 1997). Este bajo rendimiento académico hace que se aumente el tiempo efectivo de los estudiantes en la Universidad y que la duración media de las carreras sea la mitad más que su duración teórica, siendo el $50 \%$ el número de alumnos que finaliza sus estudios (Consejo de Universidades, 1997). El informe realizado por García-Varcarcel, Salvador y Zubieta (1991), señala que el 65\% de los abandonos se producen en el primer curso y que en las titulaciones técnicas, repiten curso hasta un $30 \%$ de los estudiantes de primero y que el porcentaje de alumnos presentados a las convocatorias es inferior al 30\%. Este alto nivel de fracaso académico, además de originar un elevado coste económico y social, así como altos niveles de frustración en muchos jóvenes, a su vez influye, significativamente en la calidad de la enseñanza al elevar el índice de masificación (Valle, 1997). Por otro lado, la agencia Europa Press (2001) apunta que casi cuatro de cada diez universitarios sufre depresión, aunque sólo 8 de cada 100 las sufre con frecuencia. 
Desde un punto de vista teórico, la motivación es uno de los componentes críticos del aprendizaje y a la vez uno de los más difíciles de medir. El deseo de aprender algo es el producto de muchos factores como historia previa (personalidad) y habilidades del estudiante, características de la tarea, incentivos por aprender, contexto institucional y conducta del profesor entre otros. Sí el punto de partida es que todos los estudiantes están motivados, la cuestión sería ¿para hacer qué? Las publicaciones científicas indican que el rol del educador no es incrementar la motivación per se sino descubrir, animar y sostener la motivación del estudiante para aprender y para engancharse en actividades que conduzcan al aprendizaje. Sin embargo ¿cuál es el rol de la institución en la que tienen lugar esos procesos de interacción educativa entre profesor y estudiante?, ¿debe crear condiciones favorables para que los profesores cumplan con el rol asignado?. Los niveles alarmantes de fracaso académico y abandono citados anteriormente, pueden únicamente entenderse asumiendo un fallo generalizado en la organización, planificación y puesta en práctica de las funciones formadora, investigadora y social propias de la universidad.

Definir la motivación como estado interno que incita, dirige y mantiene la conducta en el tiempo (Baron, 1998; Schunk, 1991) es sólo el comienzo, pues desde el punto de vista aplicado, la pregunta inmediata que hemos de responder es cómo generar y/o mantener esos estados internos. Sabemos que la respuesta depende del enfoque teórico que adoptemos, por lo que sí entendemos la motivación como una consecuencia del reforzamiento o la historia de reforzamiento, estaremos utilizando modelos conductuales (Chance, 1992; Strong, Silver y Robinson, 1995). Sin embargo, sí concebimos la motivación como la manifestación de una serie de necesidades básicas innatas, nuestra referencia serán los modelos humanistas de motivación (Maslow, 1968). En cambio, si pensamos en la motivación como la tendencia generalizada de luchar y buscar el éxito y elegir actividades orientadas a objetivos y a éxito y cuando se falla, redoblar los esfuerzos hasta conseguir el éxito, entonces estamos ante un enfoque cognitivo o de Motivación de Logro (McClelland y Atkinson, 1948). Entre los modelos cognitivos también podemos destacar la aproximación de Weiner (1992) y su modelo basado en las Atribuciones (Locus de Control, Estabilidad, Responsabilidad) del éxito o fracaso. O bien el modelo de las Expectativas, que sostiene que la motivación de una persona para alcanzar algo depende del producto entre la estimación que esa persona hace de sus probabilidades de obtener éxito, multiplicado por el valor que esa persona le da al éxito (Edwards, 1954; Atkinson, 1964). 
No obstante, la investigación muestra que la aparente abundancia de modelos, en realidad esconde debilidad para aislar y controlar los problemas de circularidad y direccionalidad de los efectos entre variables. Por ejemplo, algunos estudios muestran que la motivación de logro tiende a disminuir a lo largo de la escolarización, pero no está claro si es debido a la naturaleza del fenómeno o bien a las características de la educación secundaria y bachillerato (Eccles et al., 1993; Maehr y Anderman, 1993). Por otro lado, sabemos que a iguales niveles de inteligencia, obtienen mejores notas los que presentan un locus de control interno (Schunk, 1991; Shell, Colvin, y Bruning, 1995), aunque el locus de control puede cambiar y depende de la actividad o situación en cuestión, y que el locus de control influye en el rendimiento y que el rendimiento tiene un fuerte efecto en el locus de control (Weiner, 1992).

Otra aportación sumamente interesante en el campo de la motivación es la realizada por Seligman (1975) y su concepto de desesperanza (indefensión) aprendida que ocasiona déficits motivacionales, cognoscitivos y afectivos. Consiste en percibir que no importa lo que uno haga ya que está condenado a fracasar (Maier, Seligman y Solomon, 1969). La desesperanza (indefensión) aprendida puede aparecer a través de las prácticas de crianza (Hokoda y Fincham, 1995), pero también por el uso de formas impredecibles e inconscientes de reforzamientos y castigos por parte de los profesores. Aplicado a nuestro caso, los estudiantes fracasarían académicamente a través de un proceso de condicionamiento basado en feedback negativo por parte de los profesores, las experiencias escolares, los compañeros y los estudiantes mismos. Numerosos estudios muestran que cuando los estudiantes fallan de manera consistente, al final abandonarán (Seligman, 1975).

Figura 1 / Adaptación modelo de Indefensión Aprendida de Hokoda y Fincham (1995)

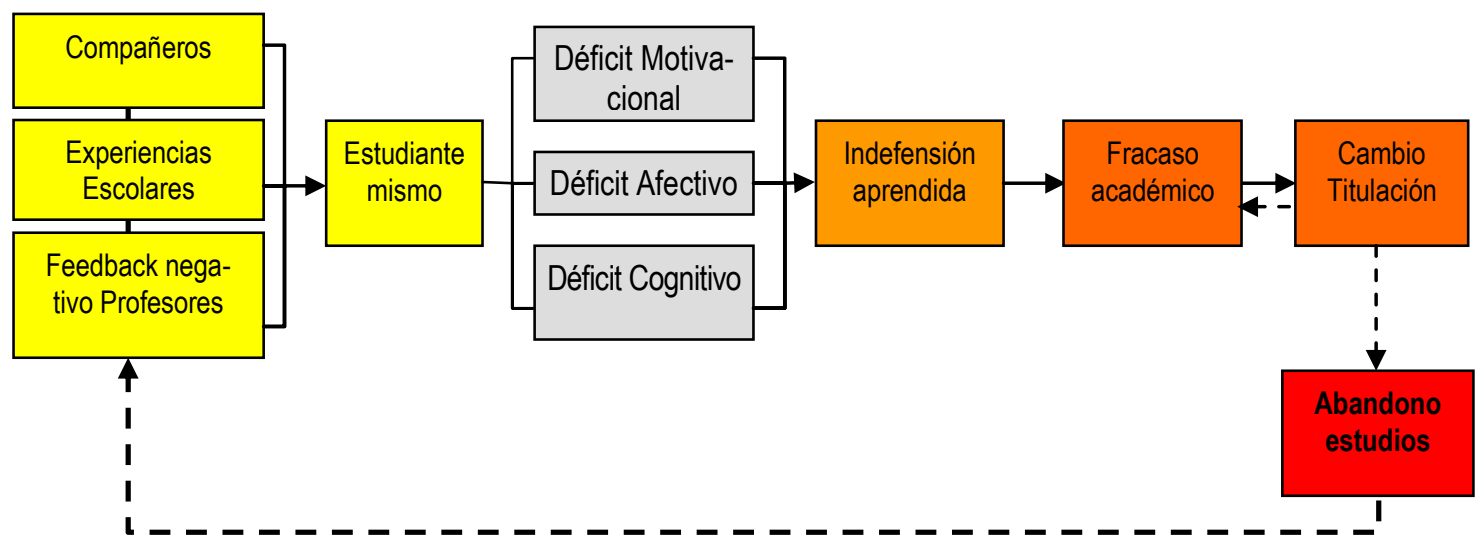


Hecha esta revisión sobre la situación que presenta la universidad española en indicadores básicos de calidad educativa, y de los modelos teóricos sobre motivación disponibles para evaluar y comprender en mayor medida qué, cómo y porqué se está produciendo esta situación, vamos a intentar comprobar la utilidad del modelo propuesto, para explicar las relaciones entre variables que influyen en la motivación y los distintos problemas de desajuste a las exigencias de la vida universitarias a los que conllevan.

\section{Método}

\section{Sujetos}

Un total de 85 estudiantes de la Universidad de Granada y usurarios del servicio de asesoramiento psicopedagógico (Gabinete Psicopedagógico, GPP), desde Enero de 2002 a Enero de 2003, componen esta muestra. La media de edad es de 23,26 años con un intervalo entre 18 y 32 años. Por sexo, el 47,06\% son mujeres y el 52,94\% hombres. El 98,82\% de los usuarios están solteros y el 1,18\% casados. Proceden de 20 Escuelas Universitarias y Facultades y 37 carreras, distribuidos desde estudiantes de primero a estudiantes de doctorado.

\section{Procedimiento}

Los estudiantes se presentan en el servicio del GPP demandando asesoramiento personal. Tras escuchar su demanda se le entrega, si procede, un protocolo de consentimiento, junto con otro protocolo en el que responden a la presencia o no de determinados problemas, dificultades o preocupaciones de índole académica, psicológica, interpersonal y vocacionalprofesional en su vida. Los 85 estudiantes completaron el Protocolo de Información Psicopedagógica (PIP), asesorados en todo momento por un profesional del centro que estuvo disponible para responder a cualquier duda o problema que les surgiera a los sujetos al aceptar las condiciones del protocolo de consentimiento, o bien al completar las áreas del PIP a las que tenían que responder. A continuación, toda la información es tabulada y registrada en una base de datos diseñada ad hoc para el servicio.

\section{Instrumentos}

El Protocolo de Información Psicopedagógica (PIP). Se trata de un inventario de 80 ítems con los que se recoge información sobre las preocupaciones y problemas que les han inducido a acudir al servicio para solicitar asesoramiento personal. Los ítems están agrupados 
en diversas áreas: demográfica, académica, psicológica, interpersonal y vocacionalprofesional. También incluye el inventario, algunas preguntas abiertas en las que los estudiantes han de describir en qué consiste su problema, los objetivos que persiguen al acudir al servicio y el nivel de esfuerzo y compromiso que están dispuestos a realizar. El inventario ha sido construido a lo largo de casi dos años, identificando las preocupaciones que presentaban los estudiantes y analizándolas en combinación con otros protocolos tomados y adaptados de otras universidades. La validez de contenido del instrumento, descansa, por tanto, en el desarrollo y revisión de la lista de temas expuestos por los estudiantes. Un método similar fue utilizado por Lubowitz (1989 citado en Nicholas, 1997) y Gallagher, Golin y Kelleher (1992) quienes construyeron su Encuesta sobre Necesidades de los Estudiantes, a partir de las preocupaciones y problemas que iban presentando los estudiantes que acudían a los servicios de Counseling durante un período de diez años.

Base de Datos en Access. Todos los registros procedentes del PIP son tabulados en esta base de datos construida ad hoc para este servicio.

\section{Análisis estadistico}

El paquete estadístico SPSS (v.11) ha sido utilizado para realizar los cálculos de los estadísticos descriptivos e inferenciales incluídos en este estudio.

\section{Resultados}

Los resultados de la Tabla 1 muestran que de los 85 sujetos que componen la población objeto el $52,94 \%$ son mujeres y el $47,06 \%$ hombres, con una media de edad de 23,26 años y un rango de 18-32 años. Según el estado civil, el 98,82\% se encuentran solteros/as y el $1,18 \%$ casados/as, y en función de la nacionalidad, el 8,24\% son extranjeros (1,18 de la UE y un 7,06 Extra UE). Si atendemos a las titulaciones y cursos de procedencia, se aprecia que la gran mayoría cursa estudios pertenecientes a las Ciencias Sociales y Jurídicas y Ciencias Experimentales y Tecnológicas (30,59\% y 27,06\%, respectivamente) y se encuentran matriculados en Cuarto (18,82\%) y Tercero (15,29\%). Por último, destacar que un 52,94\% presenta desfase académico (es decir, estar matriculados de asignaturas de varios cursos a la vez), un $23,53 \%$ ha cambiado de titulación y un $17,65 \%$ ha abandonado los estudios anteriormente. 
Tabla 1. Descripción de la muestra

\begin{tabular}{|c|c|c|c|}
\hline \multicolumn{2}{|c|}{ CARACTERÍSTICAS MUESTRA } & $\mathbf{N}$ & $\%$ \\
\hline \multirow{2}{*}{ Sexo } & Mujer & 40 & 47,06 \\
\hline & Hombre & 45 & 52,94 \\
\hline \multirow{2}{*}{ Edad } & Media & \multicolumn{2}{|c|}{23,26} \\
\hline & Rango & \multicolumn{2}{|c|}{$18-32$} \\
\hline \multirow{2}{*}{ Estado Civil } & Soltero & 84 & 98,82 \\
\hline & Casado & 1 & 1,18 \\
\hline \multirow{3}{*}{ Nacionalidad } & Española & 78 & 91,76 \\
\hline & UE & 1 & 1,18 \\
\hline & Extra UE & 6 & 7,06 \\
\hline \multirow{6}{*}{ Titulaciones } & Ciencias Sociales y Jurídicas & 26 & 30,59 \\
\hline & Humanidades & 19 & 22,35 \\
\hline & Ciencias Experimentales y Tecnológicas & 23 & 27,06 \\
\hline & Ciencias de la salud & 11 & 12,94 \\
\hline & Doctorado & 4 & 4,71 \\
\hline & Otros & 2 & 2,35 \\
\hline \multirow{10}{*}{ Cursos } & Primero & 10 & 11,76 \\
\hline & Segundo & 11 & 12,94 \\
\hline & Tercero & 13 & 15,29 \\
\hline & Cuarto & 16 & 18,82 \\
\hline & Quinto & 3 & 3,53 \\
\hline & Sexto & 1 & 1,18 \\
\hline & Varios Cursos & 21 & 24,71 \\
\hline & Terminados & 4 & 4,71 \\
\hline & Doctorado & 4 & 4,71 \\
\hline & Otros & 2 & 2,35 \\
\hline \multicolumn{2}{|r|}{ Desfase Académico } & 45 & 52,94 \\
\hline \multicolumn{2}{|r|}{ Cambios Titulación } & 20 & 23,53 \\
\hline \multirow{2}{*}{\multicolumn{2}{|c|}{ Abandono de Estudios }} & 15 & 17,65 \\
\hline & & 85 & 100,00 \\
\hline
\end{tabular}

Los resultados de los análisis estadísticos inferenciales, allí donde ha sido posible, se muestran en la Tabla 2. Podemos apreciar significatividad estadística en las variables "Titulación” que cursan y "Curso" en el que se encuentran matriculados.

Tabla 2. Resultados de la prueba Chi-Cuadrado

\begin{tabular}{|c|c|c|c|c|c|}
\hline \multicolumn{7}{|c|}{ ESTADÍSTICOS DE CONTRASTE } \\
\hline & Sexo & Titulación & Nacionalidad & Curso & Desfase \\
\hline Chi-cuadrado & 2,821 & 13,354 & 2,050 & 30,885 & 2,593 \\
gl & 1 & 3 & 2 & 9 & 1 \\
Sig. asintót. & .093 & $.004 *$ & .359 & $.000 *$ & .107 \\
\hline
\end{tabular}

${ }^{*}=p<.05$. Se ha eliminado el cero para una mayor claridad en la presentación.

Un análisis cualitativo de las respuestas emitidas por los sujetos al ser preguntados por los objetivos que persiguen al acudir al servicio, y que podríamos considerar como ejemplos 
de variables cognitivo-afectivas, académicas y sociales co-determinantes de la motivación que presentan los sujetos, nos permite agrupar sus respuestas en las siguientes categorías. Relacionadas con déficits en autocontrol, (lo cual incluye objetivos de tipo "Dejar de sentirme mal en algunos momentos", "poder cambiar mi forma de pensar", "Equilibrar los estados de ánimo", "Dejar de tener ansiedad y pasarlo mal", "Quiero que las relaciones interpersonales no me afecten", etc.) encontramos un 71,76\%; para la categoría déficit en autoestima (incluyendo respuestas del tipo "Subir mi autoestima", "Estar más a gusto conmigo misma", "Más seguridad", "Aprender a afrontar la vida con más optimismo", "Recuperar la confianza en mí", etc.) encontramos un 29,41\%; para la categoría déficit académico (que incluye objetivos como "Llevar a cabo mi carrera con éxito", "Mejorar mi rendimiento académico", "Hacer bien los exámenes, conseguir mis metas", “Terminar mi último año académico”, etc.) encontramos un porcentaje de 38,82\%; finalmente, en la categoría déficit en relaciones sociales (que incluye "Mejorar mi situación personal en relación con los demás", "Ser capaz de relacionarme sin problemas con la gente", "Mejorar habilidades y relaciones sociales", etc.) los porcentajes son del 16,47\%. Cuando los sujetos mismos evalúan los motivos de sus dificultades, un 56,47\% "Piensa que le falta motivación en los estudios", un 37,65\% afirma que "le falta motivación para seguir con la carrera", y un 10,59\% afirma que "está pensando en cambiar de titulación".

Los resultados referidos a la forma de conocer el servicio se distribuyen entre las diferentes categorías de la siguiente manera: Informados por amigos y a través de la página web acuden al servicio sendos $25,88 \%$ de los usuarios, los alumnos derivados desde otros servicios de la universidad constituyen el 9,41\%, igual porcentaje presentan las familias como canalizadoras de las demandas y las publicaciones de la universidad; el 7\% es derivado por profesores y el resto de categorías presentan otros porcentajes que aparecen detallados en la tabla 3.

Tabla 3. Formas de Conocer el GPP.

\begin{tabular}{|c|c|c|}
\hline Forma de Conocer el Servicio & $\mathbf{N}$ & $\%$ \\
\hline Amigos & 22 & 25,88 \\
\hline En otros Servicios de la Universidad & 8 & 9,41 \\
\hline Residencia & 2 & 2,35 \\
\hline Familiares & 8 & 9,41 \\
\hline Pagina Web & 22 & 25,88 \\
\hline Profesores & 6 & 7,06 \\
\hline Publicaciones de la Universidad & 8 & 9,41 \\
\hline Trípticos & 4 & 4,71 \\
\hline Poster GPP & 1 & 1,18 \\
\hline Otros & 4 & 4,71 \\
\hline Total & 85 & 100 \\
\hline
\end{tabular}


Respecto a otros indicadores directamente relacionados con la motivación con la que acuden los estudiantes al servicio, encontramos un valor medio en nivel de esfuerzo que están dispuestos a realizar de 9,45 (sobre diez). Agrupados por curso matriculado tenemos respecto a este mismo indicador 9,10 para primero, 9,68 para segundo, 9,64 para tercero, 9,53 para cuarto, 10 para quinto curso, 8,4 para los titulados; 9,53 para los que están matriculados en asignaturas de diferentes cursos; y 9 para estudiantes de Doctorado. Por titulaciones, siguiendo la tabla 4, nos encontramos que la media de motivación se distribuye de la siguiente manera: 9,41 para Humanidades; 9,80 para Ciencias Experimentales y Tecnológicas; 9,21 para Ciencias Sociales y Jurídicas; y 9,50 para Ciencias de la Salud.

Tabla 4. Nivel de Compromiso por cursos y grupos de titulaciones.

\begin{tabular}{|c|c|c|c|}
\hline \multicolumn{4}{|c|}{ NIVEL DE COMPROMISO/ESFUERZO } \\
\hline \multicolumn{4}{|c|}{ Total: 9,45} \\
\hline \multicolumn{2}{|c|}{ CURSO } & \multicolumn{2}{|l|}{ TITULACIÓN } \\
\hline 10 & 9,10 & \multirow{3}{*}{ Humanidades } & \multirow{3}{*}{9,41} \\
\hline $2^{\circ}$ & 9,68 & & \\
\hline $3^{\circ}$ & 9,64 & & \\
\hline $4^{\circ}$ & 9,53 & \multirow{2}{*}{$\begin{array}{l}\text { Ciencias Experimentales } \\
\text { y Tecnológicas }\end{array}$} & \multirow{2}{*}{9,80} \\
\hline $5^{\circ}$ & 10 & & \\
\hline $6^{\circ}$ & - & \multirow{2}{*}{$\begin{array}{l}\text { Ciencias Sociales y } \\
\text { Jurídicas }\end{array}$} & \multirow{2}{*}{9,21} \\
\hline Titulados & 8,4 & & \\
\hline Varios Cursos & 9,53 & \multirow{2}{*}{ Ciencias de la Salud } & \multirow{2}{*}{9,50} \\
\hline Doctorado & 9 & & \\
\hline
\end{tabular}

Por último, si atendemos a los datos sobre indicadores asistenciales en el Gabinete Psicopedagógico, obtenemos un total de 247 sesiones dispensadas, con una media de 2,906 sesiones por cliente. Un porcentaje del 8,18\% de cancelaciones (faltas avisando) y un 9,19\% de faltas (sin avisar). En la tabla 5 aparecen otros indicadores sobre la evaluación del servicio que hacen los usuarios en distintas categorías, así como su valoración sobre nivel de cambio en distress conseguido a lo largo del proceso de intervención.

Tabla 5. Resultados de Evaluación de Servicios del GPP .

\begin{tabular}{|l|c|c|}
\hline \multicolumn{3}{|c|}{ RESULTADOS DE EVALUACIÓN DE SERVICIOS DEL GPP } \\
\hline \multicolumn{1}{|c|}{ Áreas evaluadas } & Media $\left(^{*}\right)$ & DS \\
\hline Calidad del Servicio & 4,22 & 0,95 \\
\hline Calidad del Profesional & 4,73 & 0,51 \\
\hline Cambio Personal & 4,20 & 0,83 \\
\hline Distress Inicial & 4,43 & 0,51 \\
\hline Distress Final & 1,86 & 0,53 \\
\hline
\end{tabular}

$\left({ }^{*}\right)$ Sobre cinco puntos. 


\section{Discusión}

Hemos de tener en cuenta que cuando un estudiante acude a nuestro servicio, lo hace tras habérselo pensado, generalmente, durante bastante tiempo. Los motivos tienen que ver quizás, con la propia naturaleza del fenómeno que nos ocupa y también con la estigmatización social que todavía hoy en día conlleva acudir al psicólogo. Aún así el número de demandas es cada vez mayor, y entre los factores facilitadores de este cambio de respuesta, están por ejemplo, el hecho de poder acudir a un lugar que ofrezca este tipo de servicios de asesoramiento psicológico y/o psicopedagógico bajo ciertas condiciones de confidencialidad.

Normalmente se dice que no hay nada más práctico que una buena teoría, y entorno a esta idea va a girar nuestra discusión. Para comprobar la bondad del modelo teórico adaptado en este artículo, a partir de los trabajos de Hokoda y Fincham (1995), comenzaremos leyendo el proceso de derecha a izquierda, aunque en realidad las flechas que indican la fluctuación de las relaciones funcionales entre las variables seleccionadas, reflejan claramente la circularidad del proceso, tal y como muestra la Figura 1. Del total de los 85 sujetos atendidos y que componen esta muestra, encontramos que el 17,6\% presenta intentos anteriores de abandonos de los estudios, bien en la propia universidad, bien en niveles educativos inferiores. En la condición inmediatamente anterior de cambio de titulación encontramos un 23,5\% de los sujetos atendidos, y en la circunstancia predecesora encontramos que hasta un 53\% aproximadamente de los sujetos atendidos presenta un tipo de fracaso académico que nosotros hemos denominado "desfase académico". Este consiste en matricularse de varias asignaturas de varios cursos a la vez, lo cual da lugar en la mayoría de estos sujetos, a problemas de sobrecarga de créditos, horarios interminables, solapamiento de prácticas, etc., que se van multiplicando a medida que pasan los cursos académicos. Aunque los contrastes no paramétricos realizados (prueba chi-cuadrado) no muestran diferencias estadísticas significativas en esta variable, los alumnos atendidos bajo esta condición en nuestro servicio constituyen el grupo mayoritario tal y como refleja la tabla 1 .

Respecto a las características que presenta el problema de la falta de motivación en los sujetos atendidos, encontramos que casi un $72 \%$ de los sujetos atribuyen sus dificultades a déficits en autocontrol y casi un 30\% lo atribuye a problemas de falta de autoestima. La importancia de estos datos reside en el hecho de que tal y como pronostica el modelo, los sujetos presentan no sólo déficit en motivación, sino también déficits en otras áreas básicas de su 
comportamiento como son las cogniciones que genera y mantiene para explicar su situación y los juicios de valor que añade a esas cogniciones respecto a su capacidad o falta de ella para afrontar con éxito las demandas que su situación académica le plantea. El interés adicional de esta información reside en la proximidad de esta explicación con las de otros autores tan relevantes como Bandura (1986), al proponer su concepto de Autoeficacia como el factor (con función causal) que precede a la conducta y explicarlo como las cogniciones o verbalizaciones que el sujeto se dice a sí mismo respecto a su capacidad o no para realizar cierta conducta. Y también resulta especialmente interesante esta aproximación al fenómeno, pues enlazan con las formulaciones más recientes sobre el aprendizaje autorregulado y su potencia y utilidad para abordar este tipo de desafíos educativos. Para otro porcentaje importante de casi el $40 \%$ los déficits son también académicos en tanto en cuanto focalizan en su situación académica la fuente de sus problemas. Finalmente, para otro 17\% aproximadamente, las relaciones con los demás constituyen otro factor generador de desequilibrio en sus vidas. Los porcentajes anteriores suman más de cien pues efectivamente, tal y como habrá sospechado el lector, muchos de los sujetos señalan varias de estas circunstancias deficitarias como barreras concomitantes para alcanzar sus objetivos. Los déficits en motivación que apuntaba el modelo se confirman al señalar un 56,47\% de los sujetos atendidos que "Piensa que le falta motivación en los estudios", un 37,65\% afirma que "le falta motivación para seguir con la carrera", y un 10,59\% manifiesta que "está pensando en cambiar de titulación. Esto resulta especialmente preocupante pues no hemos de olvidar, que algunos de ellos ya han utilizado este "comodín" antes. Quizás en este punto resulte difícil mantener la asunción inicial de algunos autores de que el problema de la motivación tiene que ver más con mantenerla y orientarla que con generarla

Si atendemos al nivel de compromiso manifestado al inicio de la atención personal / académica / vocacional-profesional que se le dispensa en el GPP, encontramos niveles muy altos para todos los cursos, con el máximo nivel para el curso quinto, justo al borde de finalizar sus estudios y el nivel mínimo para el curso primero. Datos estos esperables de acuerdo a las condiciones académicas en las que se encuentran unos y otros. Analizados los datos por cursos encontramos diferencias estadísticamente significativas, por lo que podemos afirmar que la proporción de alumnos atendidos por cursos en nuestro centro es diferente a lo que cabría esperar en función de los alumnos matriculados (por cursos) en el total de la Universidad. Igual sucede al comparar los alumnos atendidos por ramas o grupos de titulaciones, donde encontramos que hay diferencias significativas. Es decir, que el número de estudiantes de las distintas ramas o titulaciones que buscan asesoramiento personal (psicológico, académico, 
vocacional) en el GPP, es diferente al que cabría esperar en función del número total de alumnos matriculados en dichas ramas o grupos de titulaciones. Lo cual se puede interpretar como que los estudiantes con necesidades y problemas académicos y motivacionales no se distribuyen por igual entre todas las titulaciones, o bien, que las circunstancias que generan déficits o dificultades de adaptación no se reparten de manera homogénea entre las diferentes titulaciones de la universidad.

Los datos adicionales que disponemos respecto a las variables que aparecen más a la izquierda del modelo, resultan especialmente significativas. Representan para muchos de estos sujetos, la posibilidad de romper la circularidad del problema que advertíamos al inicio. Efectivamente, el conocimiento sobre eficacia de intervenciones en el ámbito médico, social o psicológico recomienda intervenir los problemas en sus fases más tempranas posibles, ya que las probabilidades de éxito de una intervención generalmente son directamente proporcionales al momento de desarrollo del problema en el que se intervenga, es decir, más éxito cuanto más tempranamente. De ahí que los valores de las variables más a la izquierda del modelo sean especialmente importantes. En este sentido, que un profesor en lugar de aportarle feedback negativo a un alumno, se interese por su situación académica y lo derive a los servicios oportunos (por ejemplo al Gabinete Psicopedagógico de la universidad), significa por un lado, inhibir y/o invertir la influencia negativa de ese factor en el proceso de desmotivación del alumno. Y por otro, que el profesor se interesa por cumplir adecuadamente sus funciones tutoriales en la universidad y/o que mantiene una escucha-búsqueda activa en su entorno profesional, pues conoce la existencia del servicio. Y efectivamente este es el caso para un $7 \%$ de los alumnos recibidos en nuestro centro, pues han sido derivados por profesores. Respecto al segundo conjunto de factores referidos como experiencias escolares previas, encontramos en la misma línea argumental que hemos utilizado para la variable anterior referida al profesorado, que casi un $10 \%$ de los usuarios han sido derivados por otros servicios de la universidad y un porcentaje similar supone los que han sido aconsejados por algún familiar. En cuanto a la variable amigos, el modelo de nuevo parece acertar pues hasta casi un $26 \%$ de los demandantes han conocido el servicio a partir de sus compañeros y amigos, lo cual nos confirma la importancia de la red social de apoyo, a la hora de predecir el ajuste a su nueva vida, o al menos las probabilidades de conocer y/o acceder a servicios que puedan necesitar. Nuestros datos revelan en cambio otro aspecto importante y novedoso, desde el punto de vista de conocer y acceder al servicio (como vía alternativa o complementaria a la red social), como es la página Web, pues esta supone igualmente otro $26 \%$ aproximadamente de los ingresos. 
Finalmente, nos gustaría reflexionar sobre algunos datos disponibles sobre la evaluación que del servicio recibido realizan los estudiantes, pues entendemos que tienen un efecto significativo precisamente sobre los niveles de motivación que disponen para hacer frente a sus dificultades académicas y, por extensión, personales. De acuerdo a nuestros datos, los niveles de distress inicial comparados con los que manifiestan al finalizar la intervención son considerablemente más bajos (4,43 frente a 1,86 , sobre un total de cinco), con lo que contribuimos a reducir las probabilidades de fracaso y/o abandono, al mejorar los niveles de autocontrol y por ende de autoestima que los sujetos disponen para hacer frente a las demandas de distinto tipo, no sólo académicas de su entorno. Otros indicadores indirectos de la fiabilidad y validez de los datos sobre nivel de distress, son por ejemplo el bajo porcentaje de cancelaciones $(8,18 \%)$ y faltas $(9,19 \%)$ que presenta el servicio, lo cual refleja igualmente el uso responsable que los estudiantes pueden hacer de los servicios cuando estos se ajustan de verdad a sus necesidades.

\section{Conclusiones}

En este momento, nuestras posibilidades como científicos y educadores de controlar el fenómeno de la motivación y sus efectos en los procesos de aprendizaje y de desarrollo, está supeditado a la diversidad de modelos disponibles y a la interdependencia entre las distintas variables identificadas. Es decir, estamos ante un fenómeno extraordinariamente complejo y que el proceso se retroalimenta a sí mismo.

Para resolver el problema de la desesperanza aprendida hemos de trabajar desde distintos frentes. Desde el plano institucional, (a) desarrollando programas que ayuden a los docentes a revisar sus métodos de presentación dando por ejemplo feedback más inmediato, o manteniendo expectativas positivas y consistentes hacia sus alumnos, (b) también mejorando los materiales que utilizan, de manera que incrementen y mantengan el interés y la curiosidad de sus estudiantes, y además (c) incrementar y mejorar la oferta de servicios de asesoramiento y orientación dentro de las propias universidades como ocurre con el Gabinete Psicopedagógico de la UGR (www.ugr.es/local/ve/gpp.html). Desde el plano curricular, mediante programas que enseñen a los estudiantes a establecer sus propios objetivos de aprendizaje, a entrenarles en atribución y reestructuración de objetivos, a darse retroalimentación a sí mismos y a aplicarse premios y castigos en las condiciones y en la magnitud apropiadas. 
Pero todo esto ha de hacerse antes de que ingresen en la universidad, tanto en la Educación Secundaria como, especialmente, en la Educación Primaria, pues siguiendo el principio de prevención o intervención temprana comentado anteriormente, es entonces cuando más margen de éxito tendremos.

\section{Referencias}

Arco, J.L. y Fernández-Balboa, J.M. (2003). Barriers to School Reforms in Spain. International Review of Education. 46 (6), 580-600.

Atkinson, J.W. (1964). An introduction to motivation. Princeton, NJ: Van Nostrand.

Bandura, A. (1986). Social foundations of thought and action: A social-cognitive theory. Englewood Cliffs, NJ: Prentice-Hall.

Baron, R.A. (1998). Psychology (4th ed.). Boston: Allyn \& Bacon.

Bricall, J. (2000). Universidad 2000. Madrid: CRUE.

Chance, P. (1992). The rewards of learning. Phi Delta Kappan, 74 (3), 200-207.

Consejo de Universidades (1997). Informe sobre la evaluación de la calidad de las universidades. Madrid: Secretaría General.

Eccles, J.S., Wigfield, A., Midgley, C., Reuman, D., MacIver, D., y Feldlaufer, H. (1993). Negative effects of traditional middle schools on stuents'motivation. The Elementary School Journal, 93 (5), 553-574.

Edwards, W. (1954). The theory of decision making. Psychology Bulletin, 51, 380-417.

Europa Press (29/06/2001). Casi cuatro de cada diez universitarios sufren depresión, según una encuesta de la complutense. Disponible en http://www.psiquiatria.com. (acceso octubre 2002).

Gallagher, R.P., Golin, A. y Kelleher, K. (1992). The personal, career and learning skills needs of college students. Journal of College Student Development, 33, 301-309.

García-Varcarcel, A., Salvador, L. y Zubieta, J.C. (1991). Elementos para un análisis evaluativo de la Universidad. El caso de la Universidad de Cantabria. Actas de las Jornadas "La Investigación Educativa sobre la Universidad". Madrid: CIDE.

Hokoda, A., y Fincham, F.D. (1995). Origins of children's helpless and mastery achievement patterns in the family. Journal of Educational Psychology, 87, 375-385.

Ley de la Reforma Universitaria (LRU) (1983). Ley Orgánica 11/1983 de 25 de Agosto de Reforma Universitaria. (Publicada en BOE no 209, de 1 de Septiembre de 1983). Disponible en: http://www.upv.es/perfiles/legisla/lruc.html (acceso Enero 2004) 
Ley Orgánica de Universidades (LOU) (2001). Ley Orgánica 6/2001 de 21 de Diciembre de Universidades. (Publicada en BOE n ${ }^{0}$ 307, 24 de Diciembre de 2001). Disponible en http://www.boe.es/boe/dias/2001-12-24/seccion1.html (acceso Enero 2004).

Maehr, M.L., y Anderman, E.M. (1993). Reinventing schools for early adolescents: Emphasizing task goals. The Elementary School Journal, 93 (5), 593-610.

Maier, S.F., Seligman, M.E.P., y Solomon, R.L. (1969). Pavlovian fear conditioning and learned helplessness. En B.A. Campbell y R.M. Church (Eds), Punishment and adverse behaviour. New York: Appleton-Century-Crofts.

Maslow, A.H. (1968). Toward a psychology of being (2 $2^{\text {nd }}$ ed.). New York: Van Nostrand Reinhold.

McClelland, D.C., y Atkinson, J.W. (1948). The projective expression of needs: II. The effect of different intensities of the hunger drive on thematic apperception. Journal of Experimental Psychology, 38, 643-658.

Nicholas, L. (1997). Patters of utilization of university counseling services: a comparison of a South African and a North American University. International Journal for the Advancement of Counseling, 19, 65-71.

Schunk, D. (1991). Self-efficacy and academia motivation. Educational Psychologist, 26, 207-231.

Seligman, M.E.P. (1975). Helplessness: On depression, development, and death. San Francisco: Freeeman.

Shell, D.F., Colvin, C., y Bruning, R.H. (1995). Self-efficacy attribution and outcome expectancy mechanism in reading and writing achievement: Grade level and achievementlevel differences. Journal of Educational Psychology, 87, 386-398.

Strong, R., Silver, H.F. y Robinson, A. (1995). What do students want (and what really motivates them)? Educational Leadership, 53 (1), 8-12.

Valle, A. (1997). Determinantes cognitivo-motiacionales del rendimiento académico en estudiantes universitarios. Tesis Doctoral. Universidade da Coruña.

Vidal, J., Díez, G. y Vieira, M.J. (2001). La oferta de los servicios de orientación en las universidades españolas: innovación y ajuste a las necesidades de la comunidad universitaria. Informe final del Proyecto. Acceso en octubre de 2002 en la Universidad de León, Departamento de Filosofía y Ciencias de la Educación (Área MIDE) http://www3.unileon.es/dp/amide/inves/publi/Servori.pdf

Weiner, B. (1992). Human motivation: Metaphors, theories, and research. Newbury Park, CA: Sage. 